\title{
Field-induced thermal response and irreversible phase transition enthalpy change in $\mathrm{Pb}\left(\mathrm{Mg}_{1 / 3} \mathrm{Nb}_{2 / 3}\right) \mathrm{O}_{3}-\mathrm{PbTiO}_{3}$
}

\author{
J. Peräntie, ${ }^{\text {a) }}$ J. Hagberg, A. Uusimäki, and H. Jantunen \\ Microelectronics and Materials Physics Laboratories and EMPART Research Group of Infotech Oulu, \\ University of Oulu, P.O. Box 4500, FIN-90014 Oulu, Finland
}

(Received 5 February 2009; accepted 21 February 2009; published online 12 March 2009)

\begin{abstract}
In order to determine the enthalpy change in an irreversible field-induced phase transition, the thermal response to an electric field in $0.87 \mathrm{~Pb}\left(\mathrm{Mg}_{1 / 3} \mathrm{Nb}_{2 / 3}\right) \mathrm{O}_{3}-0.13 \mathrm{PbTiO}_{3}$ was measured and dismantled into characteristic dielectric hysteresis, reversible electrocaloric, and irreversible phase transition responses. Below the depolarization temperature $T_{\mathrm{dp}}=18{ }^{\circ} \mathrm{C}$, the phase transition enthalpy change increases rapidly to a maximum value of $|\Delta H|=77 \mathrm{~J} / \mathrm{kg}$. Above $T_{\mathrm{dp}}$, the field-induced thermal response shows a reversible nature with an increased electrocaloric effect. In addition to earlier enthalpy data presented for temperature-induced transitions, this letter provides information on the enthalpy change in a field-induced phase transition of relaxor ferroelectrics. (C) 2009 American Institute of Physics. [DOI: 10.1063/1.3098067]
\end{abstract}

Among other perovskite relaxor ferroelectrics, lead magnesium niobate $\mathrm{Pb}\left(\mathrm{Mg}_{1 / 3} \mathrm{Nb}_{2 / 3}\right) \mathrm{O}_{3}(\mathrm{PMN})$ characteristics are attributed basically to the existence of lattice disorder and polar nanoregions in a highly polarizable lattice. ${ }^{1,2}$ As a distinction from traditional ferroelectrics, material exhibits only a short-range macroscopical order in the average cubic relaxor lattice, ${ }^{3}$ and a ferroelectric-like long-range macroscopic order can develop through the application of an electric field of sufficient strength at low temperatures. ${ }^{4,5}$ In a solidsolution of $(1-x) \mathrm{Pb}\left(\mathrm{Mg}_{1 / 3} \mathrm{Nb}_{2 / 3}\right) \mathrm{O}_{3}-x \mathrm{PbTiO}_{3}(\mathrm{PMN}-x \mathrm{PT}$, $x$ in mol \%), an increasing portion of ferroelectric $\mathrm{PbTiO}_{3}$ also tends to increase the size of the polar regions and their interaction, leading to the formation of macrodomain states. ${ }^{6}$ At low $x$, PMN- $x$ PT behaves like a canonical relaxor PMN and has a mixed phase of an average cubic lattice with confined rhombohedral distortion, ${ }^{7,8}$ and on this account, an external stimulus is needed to induce a macroscopic ferroelectric order. The nature of a field-induced transition from nonergodic relaxor to the ferroelectric phase is considered to be irreversible, ${ }^{9}$ and the formed polar phase can be relaxed with substantial zero-field heating to above the intrinsic $\mathrm{Cu}$ rie temperature $T_{C 0}$, which is in principle equivalent to the zero-field thermal depolarization temperature $T_{\mathrm{dp}}$. Additionally, the ferroelectric phase can be induced at temperatures above, but close to, $T_{C 0}$, which is indicated by the fielddependent Curie temperature $T_{C}(E)$, and in that case the material shows a behavior similar to that of ordinary ferroelectrics with a first-order phase transition. ${ }^{5,10}$ However, in contrast to a transition at lower temperatures, the fieldinduced ferroelectric phase appearing above $T_{C 0}$ at temperatures just above the $T_{C}(E)$ line has a metastable nature, and thus the phase can switch reversibly back to nonpolar in the absence of the inducing field. ${ }^{5}$

A first-order field-induced transition to the ferroelectric rhombohedral phase is accompanied by an enthalpy change $\Delta H$, which, under adiabatic conditions, appears as a corresponding temperature change. Due to the irreversibility of the field-induced phase transition below $T_{\mathrm{dp}}$, the enthalpy

${ }^{a)}$ Electronic mail: jani@ee.oulu.fi. change as well as simultaneous reversible electrocaloric and dielectric hysteresis effects can be distinguished from the thermal response to sequentially applied electric field pulses. In this letter, the field-induced thermal response of a relaxor ferroelectric PMN-13PT polycrystalline bulk was studied in detail, and the enthalpy change in a field-induced phase transition was determined at various temperatures. Previously, specific heat anomalies related to phase transitions in the PMN- $x$ PT system have been measured by means of calorimetry for both PMN (Ref. 11) and PMN-PT (Ref. 12) crystals. In this method, the measurement is performed by heating or cooling with a constant electric field, and the enthalpy change can be extracted indirectly. Specific heat anomalies with a constant field basically indicate a temperature-induced transition at $T_{C}(E)$ instead of a field-induced transition at constant temperatures.

Preparation of the perovskite PMN-13PT ceramic sample and the measurement setup have been introduced previously. ${ }^{13,14}$ Initially, the dielectric hysteresis loop pair was measured subsequently at predefined temperatures with a maximum field of $E_{\max }=12 \mathrm{kV} / \mathrm{cm}$. Then, at each temperature, the sample was sequentially polarized first with a positive electric field pulse and after a 1 min settling period with a reversed electric field pulse $6.2 \mathrm{~s}$ long with a rise/fall time of $0.25 \mathrm{~s}$, and the corresponding thermal response was measured. Before each measurement the sample was heated to $T=100{ }^{\circ} \mathrm{C}$ in order to depolarize the sample to a virgin state.

Figure 1 shows typical thermal responses of PMN-13PT to sequential dc field pulses below the depolarization temperature. When an electric field pulse larger than the fieldinducing threshold field $E_{\mathrm{th}}$ is triggered in the virgin relaxor state, a phase transition to the ferroelectriclike polar phase takes place. Figure 1(a) shows the temperature response of a virgin-state sample where the temperature increase in an irreversible exothermic phase transition is accompanied by additional heat formation due to dielectric hysteresis and reversible electrocaloric effect (ECE). In the case of an induced polarization reversal from the remanent state $+P_{r}$ to $-P_{r}$ [Fig. 1(b)], the material is initially cooled due to the opposite direction of the field (not seen in Fig. 1 due to the 


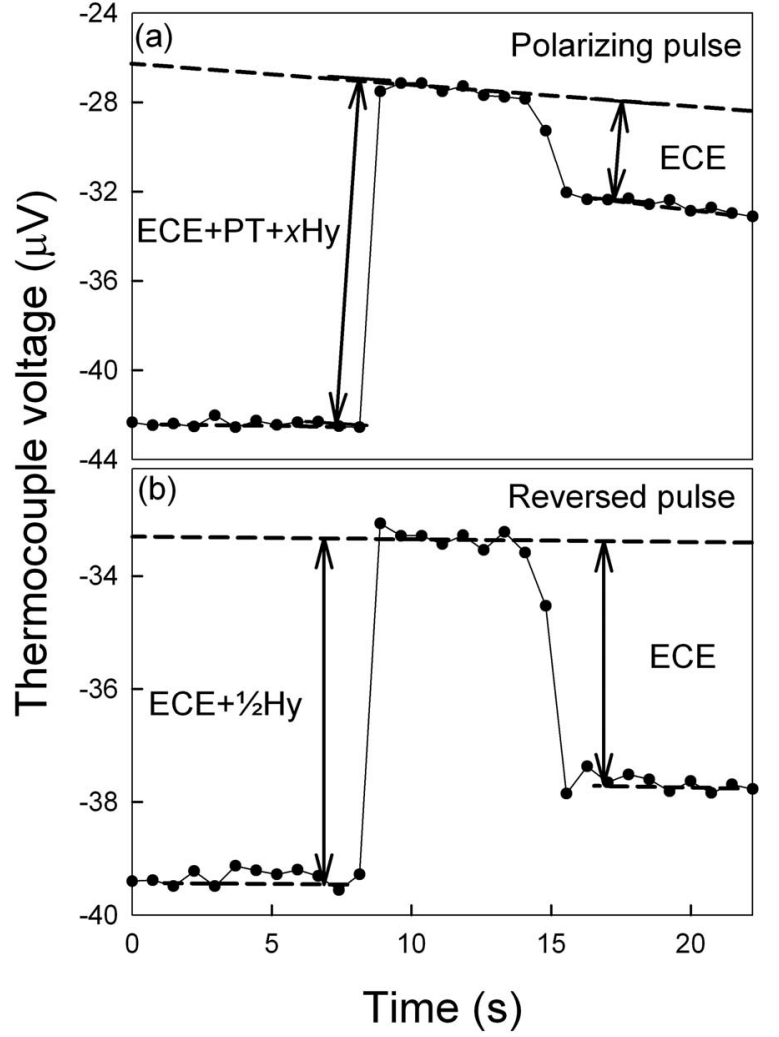

FIG. 1. Typical thermal response to polarizing and following reversed electric field pulses $\left(E=12 \mathrm{kV} / \mathrm{cm}\right.$ at $\left.T=0{ }^{\circ} \mathrm{C}\right)$ below the depolarization temperature $T_{\mathrm{dp}}$. Temperature change-related heat sources are marked with their abbreviations as follows: electrocaloric effect (ECE), initial hysteresis $(x H y)$, polarization switching hysteresis $(1 / 2 \mathrm{Hy})$, and irreversible ferroelectric phase transition $(\mathrm{PT})$.

fast field rise time). However, when the coercive field $E_{C}$ is exceeded, polarization switching begins and the thermal response consists of hysteresis and ECE. After field removal, the temperature of the sample is decreased by electrocaloric cooling. These two field pulses together with dielectric hysteresis measurements were used finally to identify the response of each participating process from the measured data.

The temperature rise caused by the initial hysteresis loss related to the virgin-state polarization $(x H y)$ was evaluated from the dielectric hysteresis loop area between the virgin curve and the $P$-axis (Fig. 2) measured at $f=0.025 \mathrm{~Hz}$. The specific heat value was approximated to be independent of temperature, with a value of $C_{p}=0.3 \mathrm{~J} / \mathrm{g} \mathrm{K}$, which was originally measured for PMN-29PT at room temperature. ${ }^{12}$ Actually, as can be seen in the case of $\mathrm{Pb}(\mathrm{Zr}, \mathrm{Ti}) \mathrm{O}_{3},{ }^{15}$ some minor hysteresis loss heating is present when the field is cycled between the maximum and remanent polarization states. Above $T_{\mathrm{dp}}$ the material is macroscopically depolarized, the irreversible field-induced phase transition no longer exists, and the temperature response is mainly caused by the ordinary ECE, dominated by the contribution of the reversible field-induced phase transition.

The measured response was dismantled into the characteristic thermal responses of all the participating effects, and these results are presented in Fig. 3 as a function of temperature for a pulse of magnitude $E=12 \mathrm{kV} / \mathrm{cm}$. Due to the reversible field-induced phase transition to the ferroelectric phase, the electrocaloric temperature change $\Delta T_{\mathrm{ECE}}$ displays a marked peak at around the depolarization temperature of

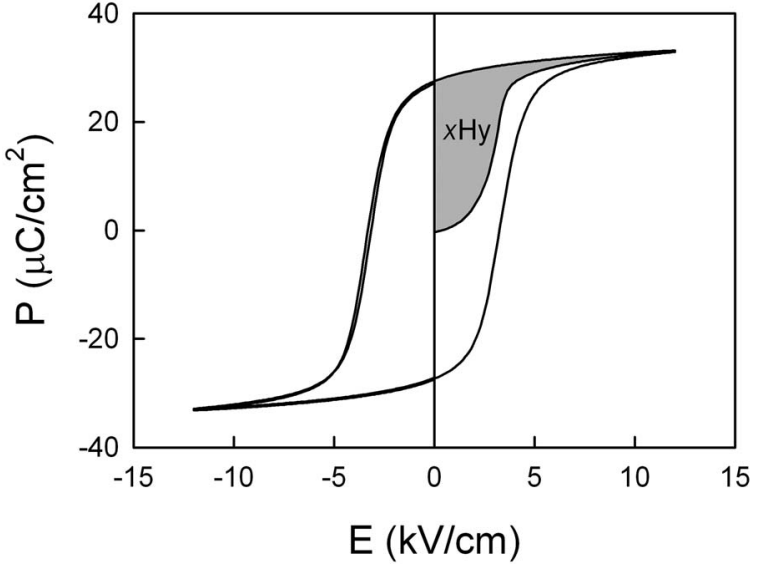

FIG. 2. Typical hysteresis loop pair measured below $T_{\mathrm{dp}}$ for the evaluation of hysteresis losses. The first loop starting from the zero polarization virgin state gives the initial hysteresis loss $(x H y)$. The switching hysteresis loss $(1 / 2 \mathrm{Hy})$ corresponds to half of the full second loop area.

$T_{\mathrm{dp}}=18{ }^{\circ} \mathrm{C}$, which was shown in Ref. 13. However, at lower temperatures $\Delta T_{\mathrm{ECE}}$ drops rapidly to below $0.1{ }^{\circ} \mathrm{C}$ when the ferroelectric phase is irreversibly induced. Below the zerofield depolarization temperature $T_{\mathrm{dp}}$, the phase transition temperature change $\Delta T_{\mathrm{PT}}$ increases rapidly from zero to a broad maximum value of $0.25{ }^{\circ} \mathrm{C}$ and decreases monotonically at lower temperatures. It is noteworthy to see that the electrocaloric temperature change $\Delta T_{\mathrm{ECE}}$ above $T_{\mathrm{dp}}$ coincides with the summed temperature response $\Delta T_{\mathrm{ECE}}+\Delta T_{\mathrm{PT}}$ $+\Delta T_{x H y}$ below $T_{\mathrm{dp}}$ and forms a continuous field-induced temperature response of a virgin PMN-13PT sample with a broad maximum value around $T=10^{\circ} \mathrm{C}$. Also, the dielectric hysteresis loop area of the ferroelectric phase increases toward lower temperatures, leading to an increase in induced temperature changes caused by initial polarization development $\left(\Delta T_{x H y}\right)$ and polarization switching $\left(\Delta T_{1 / 2 H y}\right)$.

Figure 4 shows a more detailed response of the induced phase transition. The temperature change is transformed into the corresponding enthalpy change per unit mass $\Delta H$ by multiplying it with the specific heat $C_{p}$, whose temperature dependence was omitted. The absolute value was used since the exothermic reaction is indicated with a negative value of $\Delta H$. The results show that a field strength of $4 \mathrm{kV} / \mathrm{cm}$ is

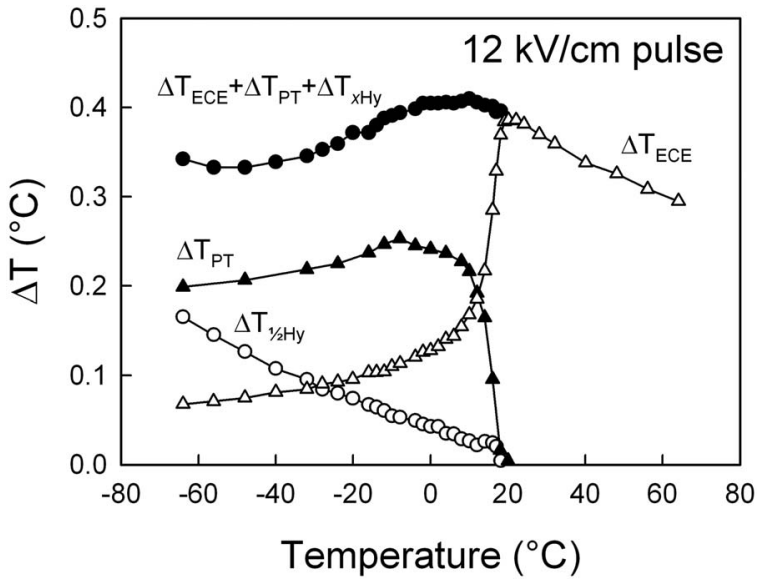

FIG. 3. Field-induced thermal response of PMN-13PT as a function of temperature. Thermal changes are assigned for irreversible ferroelectric phase transition $\left(\Delta T_{\mathrm{PT}}\right)$, reversible $\mathrm{ECE}\left(\Delta T_{\mathrm{ECE}}\right)$, and polarization formation and switching hysteresis losses $\left(\Delta T_{1 / 2 H y}\right.$ and $\left.\Delta T_{x H y}\right)$. 


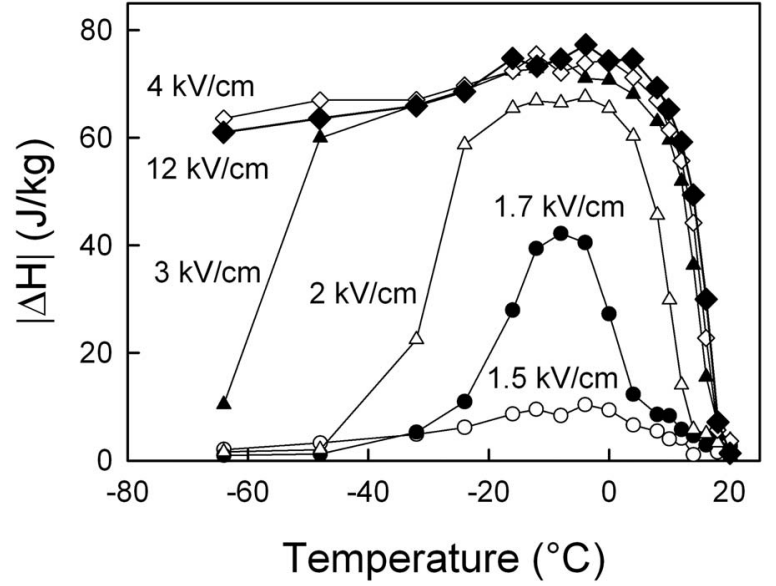

FIG. 4. Field-induced phase transition enthalpy change as a function of temperature for different electric fields.

already sufficient to fully induce a phase transition in the studied temperature range since it coincides with the response to $E=12 \mathrm{kV} / \mathrm{cm}$. At lower fields the inducement takes place only at selected temperature ranges without saturation. Initial hysteresis losses were hard to estimate at low fields due to undeveloped hysteresis loops, and therefore relative values calculated previously for well-defined hysteresis loops at $E=12 \mathrm{kV} / \mathrm{cm}$ were also used to evaluate low field losses. The absolute value of the maximum phase transition enthalpy change was found to be $|\Delta H|=77 \mathrm{~J} / \mathrm{kg}$.

When an electric field of $E=1.5 \mathrm{kV} / \mathrm{cm}$ was applied, the enthalpy change was barely $10 \%$ of its saturated value. A slightly higher field of $E=1.7 \mathrm{kV} / \mathrm{cm}$ increased $|\Delta H|$ to about half of the saturated value at a temperature range from -12 to $-4{ }^{\circ} \mathrm{C}$. A distinctive increase in $|\Delta H|$ with a small increase in the field around the threshold field shows a similar behavior as the temperature characteristics of the inducing threshold field determined by dielectric measurements. ${ }^{14}$ The extracted maximum enthalpy change of $|\Delta H|=77 \mathrm{~J} / \mathrm{kg}$ is smaller than the temperature-induced change of $|\Delta H|$ $=110 \mathrm{~J} / \mathrm{kg}$ calculated from the specific heat data measured by means of zero-field heating after field cooling for PMN. ${ }^{13}$ This difference is presumably caused by differences in the transition itself and its enforcement type as well as material composition. However, the enthalpy change values exhibit comparable magnitudes.

This letter depicted the behavior of a field-induced thermal response in PMN-13PT relaxor ferroelectric ceramic. Below the thermal depolarization temperature $T_{\mathrm{dp}}=18{ }^{\circ} \mathrm{C}$, the material temperature changed due to an irreversible fieldinduced phase transition, reversible electrocaloric ordering, and domain reorientation-related hysteresis. Above $T_{\mathrm{dp}}$ the field-induced temperature change consisted of the reversible ECE. The observed behavior of the thermal response indicates the irreversibility of a field-induced phase transition below $T_{\mathrm{dp}}$. Furthermore, the enthalpy change in an irreversible transition was calculated from the corresponding extracted temperature response, and it had a broadened peak with a maximum value of $|\Delta H|=77 \mathrm{~J} / \mathrm{kg}$ at $-4{ }^{\circ} \mathrm{C}$.

The author J.P. acknowledges the Graduate School in Electronics, Telecommunications and Automation (GETA), the Jenny and Antti Wihuri Foundation, the Tauno Tönning foundation, and the Riitta and Jorma J. Takanen foundation for financial support of the work.

${ }^{1}$ G. A. Samara, J. Phys.: Condens. Matter 15, R367 (2003).

${ }^{2}$ W. Kleemann, J. Mater. Sci. 41, 129 (2006).

${ }^{3}$ N. de Mathan, E. Husson, G. Calvarn, J. R. Gavarri, A. W. Hewat, and A. Morell, J. Phys.: Condens. Matter 3, 8159 (1991).

${ }^{4}$ H. Arndt, F. Sauerbier, G. Schmidt, and L. A. Shebanov, Ferroelectrics 79, 145 (1988).

${ }^{5}$ Z.-G. Ye and H. Schmid, Ferroelectrics 145, 83 (1993).

${ }^{6}$ Z.-G. Ye and M. Dong, J. Appl. Phys. 87, 2312 (2000).

${ }^{7}$ P. M. Gehring, W. Chen, Z.-G. Ye, and G. Shirane, J. Phys.: Condens. Matter 16, 7113 (2004)

${ }^{8}$ G. Xu, D. Viehland, J. F. Li, P. M. Gehring, and G. Shirane, Phys. Rev. B 68, 212410 (2003).

${ }^{9}$ A. A. Bokov and Z.-G. Ye, J. Mater. Sci. 41, 31 (2006).

${ }^{10}$ M. E. Lines and A. M. Glass, Principles and Applications of Ferroelectric and Related Materials (Clarendon, Oxford, 1977).

${ }^{11}$ M. V. Gorev, V. S. Bondarev, and K. S. Aleksandrov, JETP Lett. 85, 283 (2007).

${ }^{12}$ Y. Tang, X. Zhao, X. Feng, W. Jin, and H. Luo, Appl. Phys. Lett. 86, 082901 (2005).

${ }^{13}$ J. Hagberg, A. Uusimäki, and H. Jantunen, Appl. Phys. Lett. 92, 132909 (2008).

${ }^{14}$ J. Peräntie, J. Hagberg, A. Uusimäki, and H. Jantunen, Appl. Phys. Lett. 93, 132905 (2008).

${ }^{15}$ P. D. Thacher, J. Appl. Phys. 39, 1996 (1968). 